\title{
Low-Spin States From Decay Studies in the Mass 80 Region
}

\section{J. Döring, A. Aprahamian, and M. Wiescher}

Department of Physics, University of Notre Dame, Notre Dame, Indiana 46556
Neutron-deficient nuclei in the mass 80 region are known to exhibit strongly deformed ground states deduced mainly from yrast-state properties measured in-beam via heavy-ion fusion-evaporation reactions. Vibrational excitations and non-yrast states as well as their interplay with the observed rotational collectivity have been less studied to date within this mass region. Thus, several $\beta$-decay experiments have been performed to populate low-spin states in the neutron-deficient ${ }^{80,84} \mathrm{Y}$ and ${ }^{80,84} \mathrm{Sr}$ nuclei. An overview of excited $0^{+}$states in $\mathrm{Sr}$ and $\mathrm{Kr}$ nuclei is given and conclusions about shape evolution at low-spins are presented. In general, the non-yrast states in even-even Sr nuclei show mainly vibrationlike collectivity which evolves to rotational behavior with increasing spin and decreasing neutron number.

Key words: low-spin states; neutron deficient nuclei; prolate deformation.

Accepted: July 22, 1999

Available online: http://www.nist.gov/jres

\section{Introduction}

There is now extensive experimental evidence for large prolate deformation in the neutron-deficient $\mathrm{Rb}$, $\mathrm{Sr}$, and $\mathrm{Y}$ nuclei. For the even-even $\mathrm{Sr}$ isotopes, the evidence is based on experimental quadrupole moments extracted from level lifetimes [1,2] and excitation energies of the first excited yrast states [3]. In all these neutron-deficient nuclei, the underlying cause of the prolate deformation has been attributed to the population of strongly polarizing orbitals originating from the $d_{5 / 2}$ and/or intruder $g_{9 / 2}$ subshells and large gaps in the single-particle level energies.

The evolution of shapes of mass 80 nuclei from nearspherical to $\gamma$-soft and to well-deformed shapes as function of particle number and angular momentum has been investigated using different theoretical approaches [46]. In some cases, shape coexistence interpretations have been invoked to describe irregularities of the moments of inertia of some neutron-deficient even-even $\mathrm{Se}, \mathrm{Kr}$, and $\mathrm{Sr}$ nuclei at low spins [7]. For the even-even $\mathrm{Sr}$ isotopes the situation is quite complicated. Large prolate deformations as observed for ${ }^{76,78} \mathrm{Sr}$ are in agreement with most of the recent calculations while the nucleus ${ }^{80} \mathrm{Sr}$ is predicted to be spherical in the ground state with $\beta_{2}=0.053$ [6]. The ground-state deformation of $\beta_{2} \approx 0.4$ as deduced from in-beam $\gamma$-ray experiments $[1,2]$ is in contrast to recent results from fast beam laser spectroscopy [8] where the deduced mean charge radii indicate somewhat less deformed shapes for ${ }^{78,80} \mathrm{Sr}$. The neutron-deficient even-even $\mathrm{Sr}$ isotopes exhibit yrast level sequences (or moments of inertia) at low spins which show large deviations from the behavior expected for a rigid rotor, possibly indicating shape fluctuations. Thus, the issue of the rigidity of the shapes and the occurrence of co-existing configurations are not yet 
resolved and have not been thoroughly addressed as many of the key states of interest are of low spins and of non-yrast nature, i.e., they are not well populated in the heavy-ion fusion reactions usually used for the in-beam studies.

Properties of nuclei along the $N=Z$ line are also of interest for the astrophysically relevant rapid proton capture (rp) process [9] which is thought to be one of the dominant energy sources in cataclysmic binaries like novae and $\mathrm{x}$-ray bursts. The rp process is characterized by a sequence of fast proton capture reactions and subsequent $\beta$ decay. Usually, the $\beta$ decay is slow compared to the fast proton capture reactions. Waiting points can develop where the proton capture is compensated by inverse photo-disintegration or where single proton capture is inhibited at the proton-drip line. The lifetimes of these waiting-point nuclei are determined by the $\beta$ decay of the ground state or thermally excited states. Thus lifetimes of ground states and/or $\beta$-decaying isomeric states in the vicinity of the proton-drip line are important input parameters for calculations of nuclear synthesis, luminosity, and time scale [10]. Nucleosynthesis at the extreme temperature and density conditions associated with such events may well proceed beyond the doubly-magic ${ }^{56} \mathrm{Ni}[11]$.

Only few alternative probes are available for investigating non-yrast states in nuclei far from stability. The most useful is the careful investigation of the $\beta$ decay from a higher- $Z$ parent nucleus. The parent spins are usually low so a large number of non-yrast states is expected to be populated when the decay energy is large. For a successful $\beta$-decay experiment sufficient production of the parent nuclei is needed. Far from stability, this is experimentally difficult as production cross section are small and the nuclei are short-lived.

\section{Low-Lying Isomers in the Odd-Odd ${ }^{80,84}$ Y Isotopes}

\subsection{New Isomer in ${ }^{80} Y$}

A new $\beta$-decay experiment has been performed to study the low-spin structure of the $N=Z+2$ nucleus ${ }^{80} \mathrm{Y}$. The ${ }^{80} \mathrm{Y}$ source has been produced via the fusionevaporation reaction ${ }^{24} \mathrm{Mg}\left({ }^{58} \mathrm{Ni}, \mathrm{pn}\right)$ reaction at $190 \mathrm{MeV}$. The use of inverse kinematics provided a strongly forward-peaked recoil spectrum best suitable for an efficient collection and subsequent separation by the Argonne fragment mass analyzer [12]. The $A=80$ mass separated recoils were implanted on a plastic tape and transported to a $\beta$ - and $\gamma$-ray counter station consisting of three Ge detectors and a low-energy photon spec- trometer. Each $\gamma$-ray detector had a thin plastic scintillator in front for the detection of $\beta$ rays. The recoils were implanted within a deposition time of $20 \mathrm{~s}$ and their radioactive decay was subsequently measured for $20 \mathrm{~s}$. Several cycles were also performed with $60 \mathrm{~s}$ deposition time and $60 \mathrm{~s}$ counting time. More experimental details have been reported in Ref. [13].

A single $\gamma$-ray spectrum recorded with the low-energy photon spectrometer and representative for the decay of the short-lived mass 80 recoils is displayed in Fig. 1 . The strongest $\gamma$-ray peak has been identified as the $2^{+} \rightarrow 0^{+}$transition in ${ }^{80} \mathrm{Sr}$. Further, a new $\gamma$-ray transition at $228.5 \mathrm{keV}$ has been found [13] which is the second strongest line in the spectrum. This transition depopulates a new isomer in ${ }^{80} \mathrm{Y}$ with a half-life of 4.7(3) s [13]. Spin and parity of the isomer has been determined to be $1^{-}$. Thus, the isomer decays by a M3 transition to the $4^{-}$ground state. The extracted M3 transition strength is $0.78(5)$ Weisskopf units. Most interestingly, the isomer undergoes $\beta$ decay as well to low-lying states in ${ }^{80} \mathrm{Sr}$ [14], as can be seen in the decay scheme of the isomer given in Fig. 2, upper left-hand side. This conclusion has been drawn from two experimental facts: (i) The time distribution of the $2^{+} \rightarrow 0^{+} 385.9 \mathrm{keV}$ transition in ${ }^{80} \mathrm{Sr}$ does not show the expected delayed feeding by the $228.5 \mathrm{keV}$ isomeric transition (as the $4^{+} \rightarrow 2^{+}$ $594.8 \mathrm{keV}$ transition does), i.e., the time distribution can be fitted well with a single exponential decay curve. This indicates that the delayed component is canceled out. (ii) The difference spectrum between early and late time correlated events exhibits a strong $385.9 \mathrm{keV}$ transition. This spectrum is shown in Fig. 3. The spectrum has been generated by subtracting the time- $\gamma$ events of the $15 \mathrm{~s}$ to $60 \mathrm{~s}$ time range (late events) from the time- $\gamma$ events of the $0 \mathrm{~s}$ to $10 \mathrm{~s}$ range (early events). Further, events in the time range 10 to $15 \mathrm{~s}$ have been excluded (see inset of Fig. 3). For normalization, we assumed that the intensity of the $783.1 \mathrm{keV}$ line depopulating the $6^{+}$ state at $1763.7 \mathrm{keV}$ in ${ }^{80} \mathrm{Sr}$ cancels out leading to a factor of 0.68 . As a result a small intensity amount of the 594.8 $\mathrm{keV}$ line remains in the difference spectrum. This may indicate that the $1^{-}$isomeric $\beta$ decay is highly fragmented. The situation is similar to the $1^{-}$ground-state $\beta$ decay of ${ }^{76} \mathrm{Rb}$ [15]. The difference spectrum indicates, in addition to the strong $385.9 \mathrm{keV}$ transition, a weak $1350.4 \mathrm{keV}$ line. The same $1350.4 \mathrm{keV}$ transition can be seen in the sum coincidence spectrum of the 756 and $1142 \mathrm{keV}$ gates providing evidence for a level at 2492.5 $\mathrm{keV}$. This level seems to be populated in the isomeric decay only and has probably a low spin.

The $\beta$-decay branch has been estimated to be about 19(2) \%. This result has important consequences for calculations of the rp-process nucleosynthesis of ${ }^{80} \mathrm{Kr}$ 


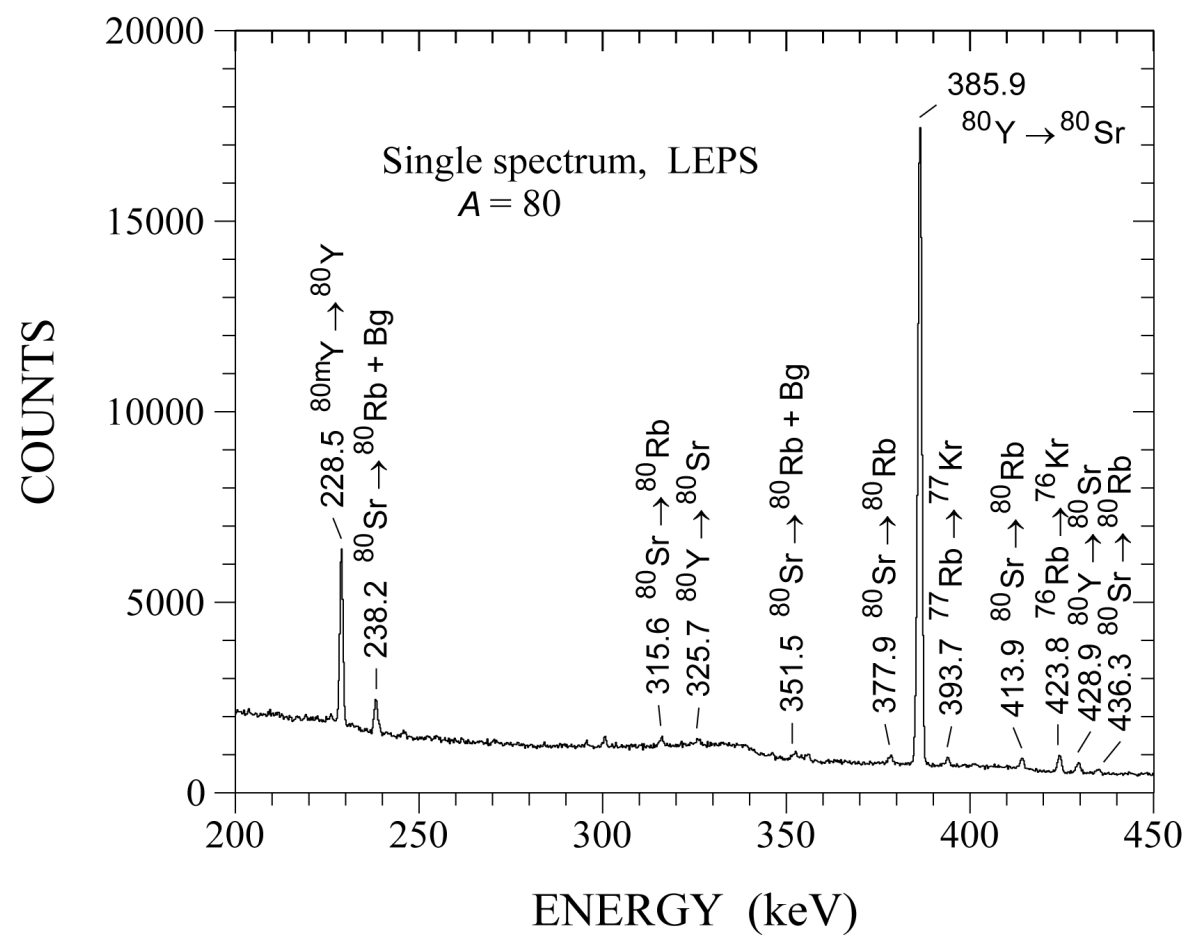

Fig. 1. Single $\gamma$-ray spectrum recorded with a low-energy photon spectrometer. The mass 80 recoils were mass separated by the Argonne fragment mass analyzer and transported to the counter station by a moving tape system. The figure has been taken from Ref. [13].

since the longer lived ground state of ${ }^{80} \mathrm{Y}\left(T_{1 / 2}=30.1(5)\right.$ $\mathrm{s}$ [13]) is partly bypassed by the isomeric $\beta$ decay, and a shorter effective half-life of ${ }^{80} \mathrm{Y}$ is obtained which leads to a reduction of the calculated overproduction of ${ }^{80} \mathrm{Kr}[10]$.

Total Routhian surface calculations [4] have shown that the odd-odd nucleus ${ }^{80} \mathrm{Y}$ exhibits a strongly deformed prolate shape with a quadrupole deformation of $\beta_{2}=0.37$ for the ground state. The prolate minimum persists up to high rotational frequencies. Thus, the deformed shape inspired the application of two-quasiparticle-plus-rotor calculations to investigate the wave functions of the low-lying states in terms of Nilsson orbitals. We found that the low-spin structure can be well explained if a proton-neutron residual interaction is employed. In this case the ordering of the states and the energy splitting between the $4^{-}$ground state and the $1^{-}$ isomer can be well reproduced. The wave functions contain mainly the proton $[422] 5 / 2^{+}$and the neutron [301]3/ $2^{-}$Nilsson orbitals. These orbitals are coupled parallel and antiparallel in the $4^{-}$ground state and in the $1^{-}$ isomer of ${ }^{80} \mathrm{Y}$, respectively. The model calculations demonstrate that the deformed picture accounts very well for the observed properties of the low-lying states in ${ }^{80} \mathrm{Y}$.

\section{$2.2 \quad$ Low-Spin States in ${ }^{84} \mathbf{Y}$}

Early evidence was presented that the odd-odd nucleus ${ }^{84} \mathrm{Y}$ has very likely an $1^{+}$ground state and a higherlying $\left(5^{-}\right)$isomer at an energy of about $500 \mathrm{keV}[16,17]$. This structure was deduced from early decay studies and the excitation energy of the isomer was an estimate only. Also, a few $\gamma$ rays had been previously assigned to the ${ }^{84} \mathrm{Zr}$ decay [18], however, not placed into a level scheme. Therefore, three new decay experiments have been carried out: (i) via the irradiation of a ${ }^{58} \mathrm{Ni}$ target with ${ }^{28} \mathrm{Si}$ ions at $97 \mathrm{MeV}$ using a modified NORDBALL setup [19], (ii) via the irradiation of a ${ }^{58} \mathrm{Ni}$ target with $99 \mathrm{MeV}$ ${ }^{28} \mathrm{Si}$ ions and (iii) via the irradiation of a ${ }^{58} \mathrm{Ni}$ target with $135 \mathrm{MeV}^{32} \mathrm{~S}$ ions [20]. The latter two experiments were performed at Florida State University. In the first two experiments the chosen target-projectile combinations ensured that the even-even nucleus ${ }^{84} \mathrm{Zr}$ was produced in-beam, without any in-beam population of states in ${ }^{84} \mathrm{Y}$ and ${ }^{84} \mathrm{Sr}$. In this way all states seen in these two latter nuclei were populated via the $\beta$-decay chain ${ }^{84} \mathrm{Zr} \rightarrow{ }^{84} \mathrm{Y} \rightarrow{ }^{84} \mathrm{Sr}$ only. The experiments at Florida State University were carried out with 5 Ge detectors and a low-energy photon spectrometer to detect the $\gamma$ rays. 


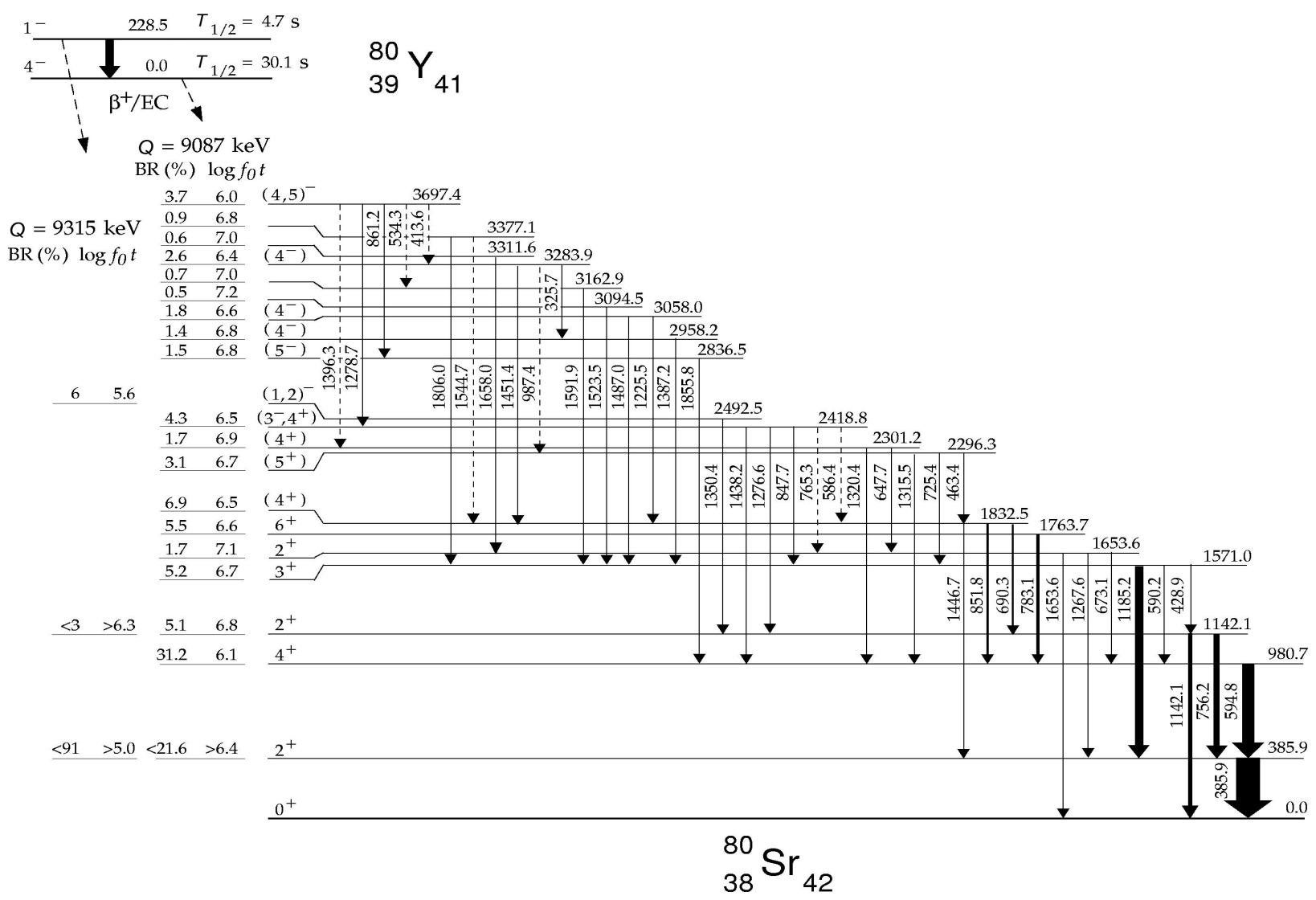

Fig. 2. Level scheme of ${ }^{80} \mathrm{Sr}$ deduced from the $\beta$ decay of ${ }^{80} \mathrm{Y}$. The figure has been taken from Ref. [14].

It has been found that the $1^{+}$isomer in ${ }^{84} \mathrm{Y}$ has an excitation energy of $67 \mathrm{keV}$ and undergoes $\beta$ decay only. No low-energy $67 \mathrm{keV} \gamma$ transition to the ground state in ${ }^{84} \mathrm{Y}$ has been seen in the singles spectrum measured with the low-energy photon spectrometer. A partial decay scheme is shown in Fig. 4 where emphasis has been placed on the low-spin structure in ${ }^{84} \mathrm{Y}$ and the population of the $0^{+}$states in ${ }^{84} \mathrm{Sr}$ by the $\beta$ decay of the $1^{+}$ isomer. Further, states up to $\left(7^{+}\right)$in the $\gamma$-vibrational band of ${ }^{84} \mathrm{Sr}$ have been identified giving evidence for a possible spin and parity assignment of $6^{+}$to the ground state of ${ }^{84} \mathrm{Y}$, in contrast to the previous assignment of $\left(5^{-}\right)$ [17].

The new decay data revealed many new $\gamma$ rays in ${ }^{84} \mathrm{Y}$ and ${ }^{84} \mathrm{Sr}$ and hence many new levels have been identified in both nuclei. For example, the previously reported excited $0^{+}$states at 1505 and $2075 \mathrm{keV}$ in ${ }^{84} \mathrm{Sr}$ as identified via a $(\mathrm{p}, \mathrm{t})$ reaction [21] have been observed via $\gamma$-ray spectroscopy at 1504 and $2072 \mathrm{keV}$, respectively, for the first time. These states depopulate via 711 and $1279 \mathrm{keV}$ transitions to the first excited $2^{+}$state at 793 $\mathrm{keV}$ in ${ }^{84} \mathrm{Sr}$. An intense $793 \mathrm{keV}$ peak has been seen only in the coincidence gates at 711 and $1279 \mathrm{keV}$ indicating a very low multiplicity. Thus the origin is very likely a low-spin state in ${ }^{84} \mathrm{Y}$, i.e., the $\beta$ decay of the $1^{+}$isomer. The number of coincidence events of the $1279 \mathrm{keV}$ line gated by the $793 \mathrm{keV}$ transition in the 10 different detector-pair matrices of experiment (iii) was good enough to deduced angular correlation coefficients [22]. They provide evidence for a $0^{+} \rightarrow 2^{+} \rightarrow 0^{+}$decay sequence.

\section{Low-Lying States in Even-Even Neutron-Deficient $\mathrm{Sr}$ and $\mathrm{Kr}$ Isotopes}

\subsection{Excited $\mathbf{0}^{+}$States in $\mathrm{Sr}$ Isotopes}

The evolution of the nuclear shape from spherical to deformed in the even-even $\mathrm{Sr}$ isotopes is well known when moving away from the neutron shell closure at $N=50$. These findings are based mainly on yrast level properties investigated via heavy-ion fusion-evaporation reactions. The study of non-yrast low-lying states may provide additional evidence to support these claims, or may indicate a more complex nuclear structure at low spins. The careful study of the $\beta$ decay of odd-odd $\mathrm{Y}$ 


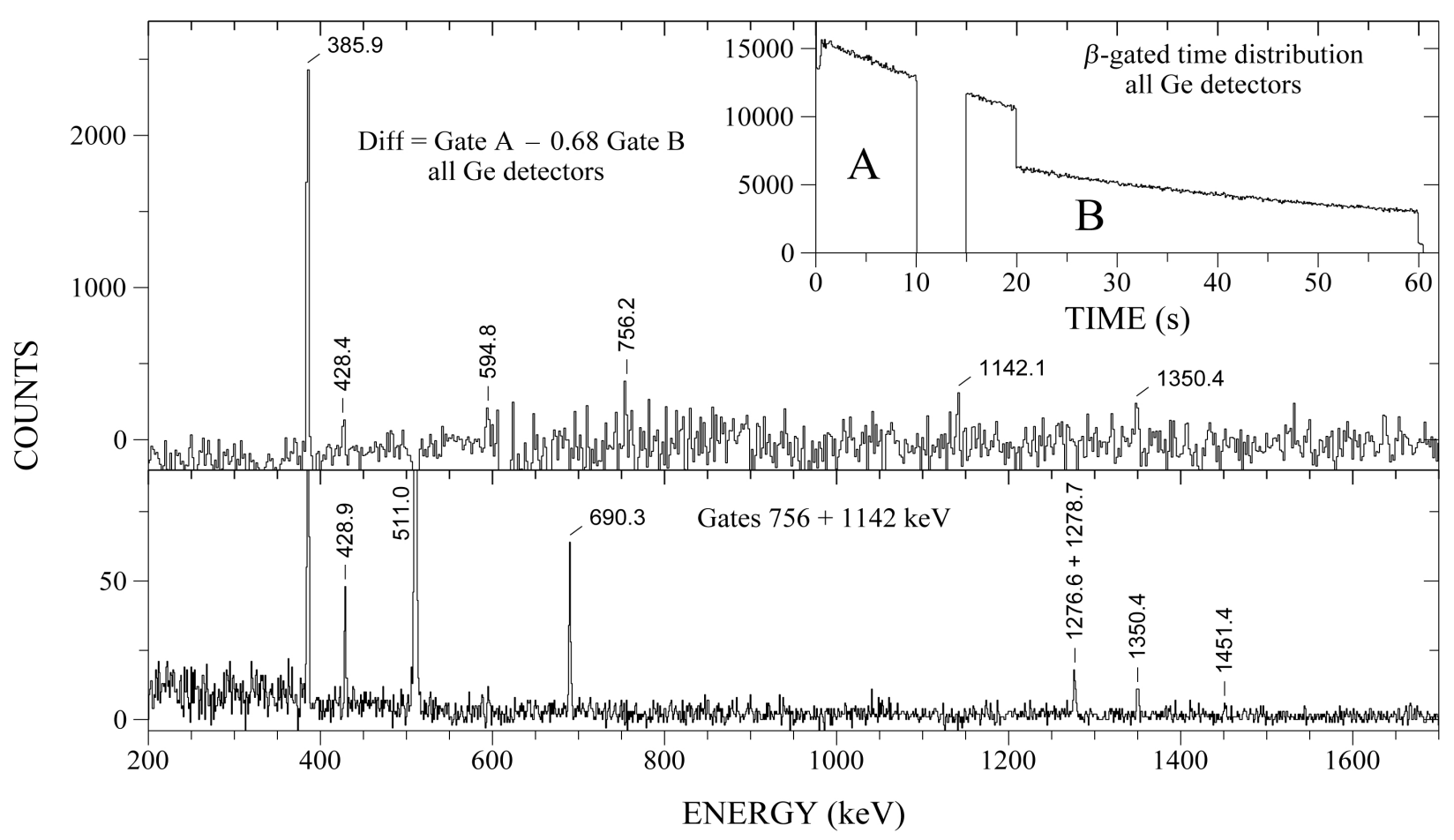

Fig. 3. Difference spectrum (top panel) of $\beta$-gated events from all Ge detectors to illustrate the decay of the $1^{-}$isomer in ${ }^{80} \mathrm{Y}$. The gating conditions are shown in the inset. To obtain the best possible statistics, the events from both $20 \mathrm{~s}$ and $60 \mathrm{~s}$ cycles have been added up causing the visible step at the time of $20 \mathrm{~s}$. The $756 \mathrm{keV}$ and $1142 \mathrm{keV}$ background-corrected sum coincidence spectrum is shown in the bottom panel. The figure has been taken from Ref. [14].

study of the $\beta$ decay of odd-odd Y nuclei seems to be the best method for populating non-yrast levels in neutrondeficient even-even $\mathrm{Sr}$ isotopes. Thus, the experiment described before for the investigation of an isomer in ${ }^{80} \mathrm{Y}$ has been analyzed for the ${ }^{80} \mathrm{Y} \rightarrow{ }^{80} \mathrm{Sr} \beta$ decay as well. The high selectivity of the Argonne fragment mass analyzer and the use of a multi-detector setup provided clean data. The known ${ }^{80} \mathrm{Sr}$ level scheme could be extended by 14 new levels [14], see Fig. 2. Spin and parity assignments are given based on the observed feeding and depopulation pattern, deduced $\log f t$ values, and on a comparison with the decay of the ${ }^{78} \mathrm{Rb} 4^{-}$isomer to low-lying states in ${ }^{78} \mathrm{Kr}$ [23].

Most of the known excited $0^{+}$states in mass 80 nuclei have been identified via radioactive decay studies or particle-transfer reactions. The experimental detection is sometimes difficult since a $0_{2}^{+} \rightarrow 0^{+} \mathrm{E} 0$ transition can be verified only via a conversion electron measurement. Using $\gamma$-ray spectroscopy, usually the $0_{2}^{+} \rightarrow 2_{1}^{+}$E2 transition is detected. In general, the E0 matrix elements depend sensitively on the nuclear charge distribution and thus on the nuclear deformation [24]. Hence, the identification of these excited $0^{+}$states in a chain of isotopes allows to study the evolution of the nuclear shape at low spins. The latest results for the even-even
Sr isotopes $(Z=38)$ are displayed in Fig. 5. The previously reported $0^{+}$states in ${ }^{84} \mathrm{Sr}$, detected via particletransfer reactions and confirmed by present $\gamma$-ray spectroscopy, are included. With decreasing neutron number, the position of the excited $0^{+}$states decreases as well and a multiplet-like grouping of the levels is obtained.

\subsection{Excited $\mathbf{0}^{+}$States in $\mathrm{Kr}$ Isotopes and $\mathbf{N}=\mathbf{3 8}$ Isotones}

The systematics of the excited $0^{+}$states in the neutron-deficient even-even $\mathrm{Kr}$ isotopes is plotted in Fig. 6 . The recently discovered low-lying $0_{2}^{+}$state in ${ }^{74} \mathrm{Kr}$, at most $85 \mathrm{keV}$ above the first excited $2^{+}$state at $456 \mathrm{keV}$ [25], refines the previously suggested shape coexistence picture [26]. This picture of a deformed-spherical shape coexistence was invoked to explain the irregularities in the energy spacings (or moments of inertias) of the lowest yrast excitations in the even-even ${ }^{74,76} \mathrm{Kr}$ nuclei. Now an oblate shape is suggested for the excited $0^{+}$state in ${ }^{74} \mathrm{Kr}$, in contrast to the prolate deformed ground-state band. The half-life reported for the $0_{2}^{+}$in ${ }^{74} \mathrm{Kr}$ is the partial time for the E0 transition. The low-energy $\gamma$-ray decay has not been found yet. 


$$
\begin{array}{r}
T_{1 / 2}=26 \mathrm{~m} \frac{0^{+}}{84 Z r} \Perp \beta^{+/ E C} \\
Q_{E C}=2700 \mathrm{keV}
\end{array}
$$
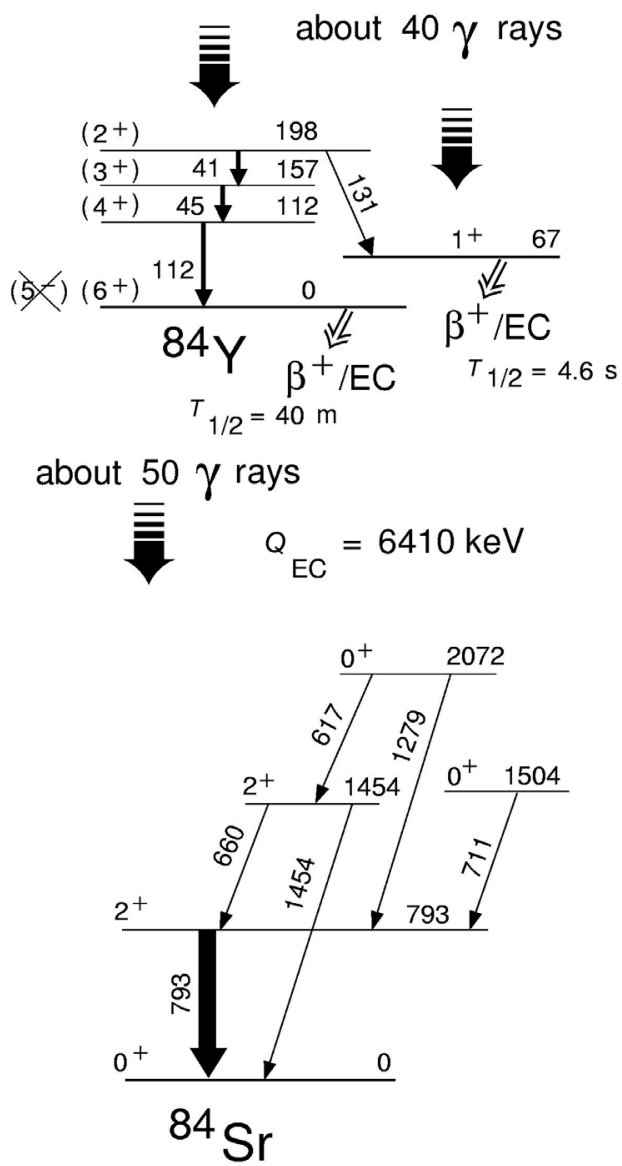

Fig. 4. Selected low-lying states in odd-odd ${ }^{84} \mathrm{Y}$ and even-even ${ }^{84} \mathrm{Sr}$ observed in $\beta$ decay via the chain ${ }^{84} \mathrm{Zr} \rightarrow{ }^{84} \mathrm{Y} \rightarrow{ }^{84} \mathrm{Sr}$ using five Ge detectors and a lowenergy photon spectrometer. The experimental results have been taken from Refs. $[19,20]$.

It should be pointed out that the second $0^{+}$state in ${ }^{74} \mathrm{Kr}$ fits quite well into the $N=38$ systematics as can be seen in Fig. 7. In most of these isotones, an excited $0^{+}$state has been found which decays by a low-energy $\gamma$ ray to the first $2^{+}$state. The deduced $0_{2}^{+} \rightarrow 2^{+} \mathrm{E} 2$ transition strengths are in the order of 45 Weisskopf units indicating substantial collectivity. The reported E0 matrix elements are also given in the figure.

\subsection{Vibration-Like Multiplets in Even-Even $\mathrm{Sr}$ Nuclei}

As can be seen in Fig. 8, the new level scheme of ${ }^{80} \mathrm{Sr}$ deduced from our $\beta$-decay study is clustered into states typical of one-, two-, and three-phonon multiplets of an anharmonic vibrational nucleus. In this approach the lowest $2^{+}$state at $385.9 \mathrm{keV}$ can be interpreted as an one-phonon vibrational state. States corresponding to the two-phonon triplet may be the observed states with spins $2_{2}^{+}$and $4_{1}^{+}$at energies of 1142.1 and $980.7 \mathrm{keV}$, respectively. From theoretical considerations there should also be a $0^{+}$state to complete the two-phonon triplet. A $0^{+}$level at $1.0 \mathrm{MeV}$ was observed in a ${ }^{78} \mathrm{Kr}\left({ }^{3} \mathrm{He}, \mathrm{n}\right){ }^{80} \mathrm{Sr}$ reaction study [29] but this level has not been seen in our decay data set. Based on a phenomenological parametrization of the effective interaction between phonons [30,31] and using experimental values for the interaction parameters as deduced from members of the observed three-phonon multiplet, a range of $820 \mathrm{keV}$ to $880 \mathrm{keV}$ can be estimated for the excitation energy of the two-phonon $0^{+}$state. For three phonons, the expected multiplet of levels consists of $0_{3}^{+}, 2_{3}^{+}, 3_{1}^{+}, 4_{2}^{+}$, and $6_{1}^{+}$. There are observed states with $2_{3}^{+}, 3_{1}^{+}, 4_{2}^{+}$, and $6_{1}^{+}$ at $1653.6 \mathrm{keV}, 1571.0 \mathrm{keV}, 1832.5 \mathrm{keV}$, and 1763.7 $\mathrm{keV}$, respectively, which might be identified with these excitations. The expected $0_{3}^{+}$level has not been seen. Similar to the estimate of the excitation energy of the $0_{2}^{+}$ state, an energy range of $1890 \mathrm{keV}$ to $2270 \mathrm{keV}$ can be deduced for the third $0^{+}$state based on the anharmonicity of the $2_{2}^{+}$state.

The observed vibrations in ${ }^{80} \mathrm{Sr}$ are clearly anharmonic since the $(2 I+1)$ weighted energy centroids of the known members of the multiplets are at $1036 \mathrm{keV}$ and $1726 \mathrm{keV}$ for $n=2$ and 3, respectively, i.e., the higher orders (with $n=2,3$ ) are not strictly a multiple of the one-phonon energy of $386 \mathrm{keV}$. The deviations from the expected energies for a harmonic vibrator can be attributed to various anharmonic effects. One such anharmonicity may arise from a finite quadrupole deformationor angular momentum dependence of the nuclear shape. Much less anharmonicity is needed to understand the low-lying states in ${ }^{84} \mathrm{Sr}$, as can be seen on the right-hand side of Fig. 8. In particular, the observed $0^{+}$states fit very well into this interpretation and complete the multiplets. The energy centroids of the $n=2$, 3 multiplets are almost a multiple of the $793 \mathrm{keV}(n=1)$ energy. Thus, an almost harmonic vibration-like nature in ${ }^{84} \mathrm{Sr}$ is deduced. 


\section{Position of excited $0^{+}$}

Even-Even $\mathrm{Sr}, \mathrm{Z}=38$
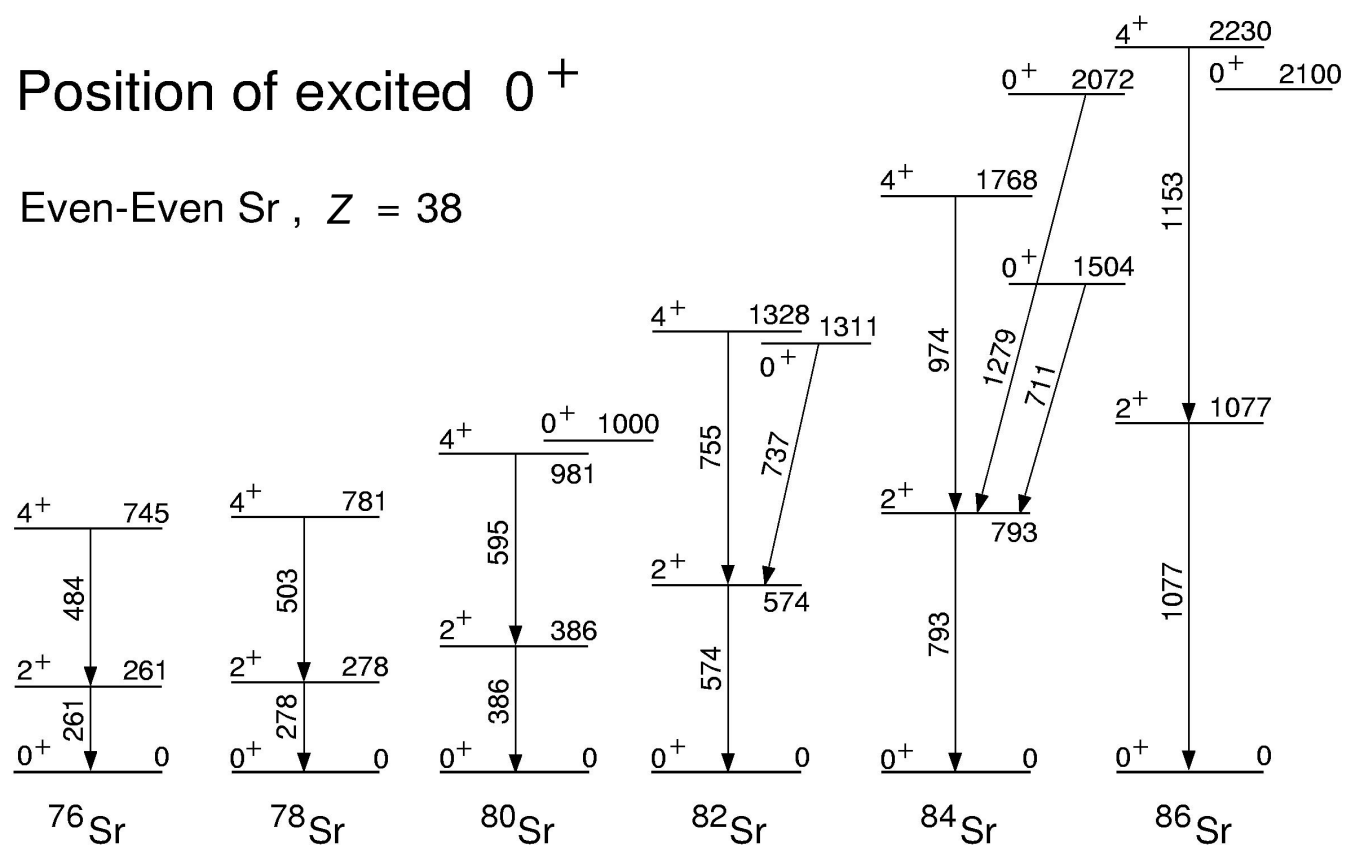

Fig. 5. Excited $0^{+}$states and the lowest yrast excitations are displayed for even-even neutron-deficient $\mathrm{Sr}$ isotopes. The experimental results on the $0^{+}$states have been taken from: ${ }^{80} \mathrm{Sr}$, Ref. [29]; ${ }^{82} \mathrm{Sr}$, Ref. [17]; ${ }^{84} \mathrm{Sr}$, Refs. [20,21]; ${ }^{86} \mathrm{Sr}$, Ref. [21].

\section{Position of excited $0^{+}$}

Even-Even $\mathrm{Kr}, \quad Z=36$
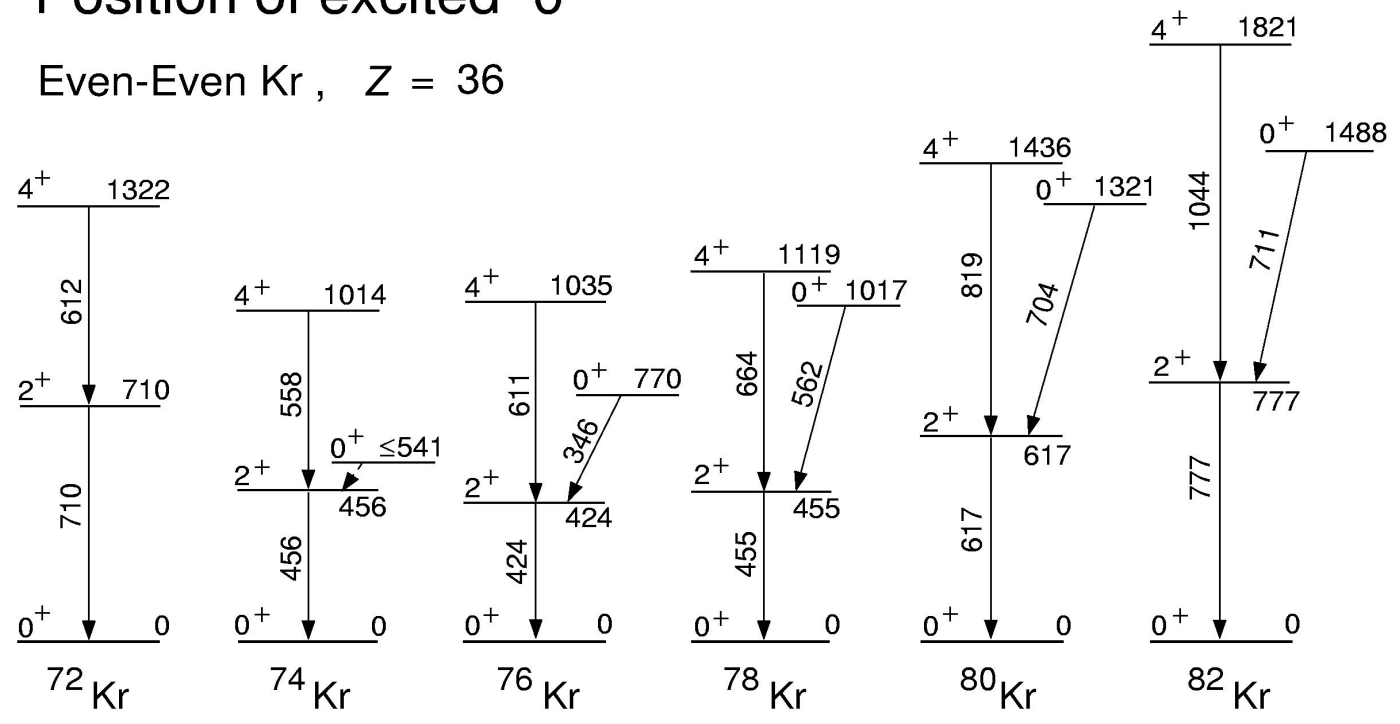

Fig. 6. Excited $0^{+}$states and the lowest yrast excitations are displayed for even-even neutron-deficient $\mathrm{Kr}$ isotopes. The experimental results on the $0^{+}$states have been taken from: ${ }^{74} \mathrm{Kr}$, Ref. [25]; ${ }^{76,78,80,82} \mathrm{Kr}$, Ref. [17]. 


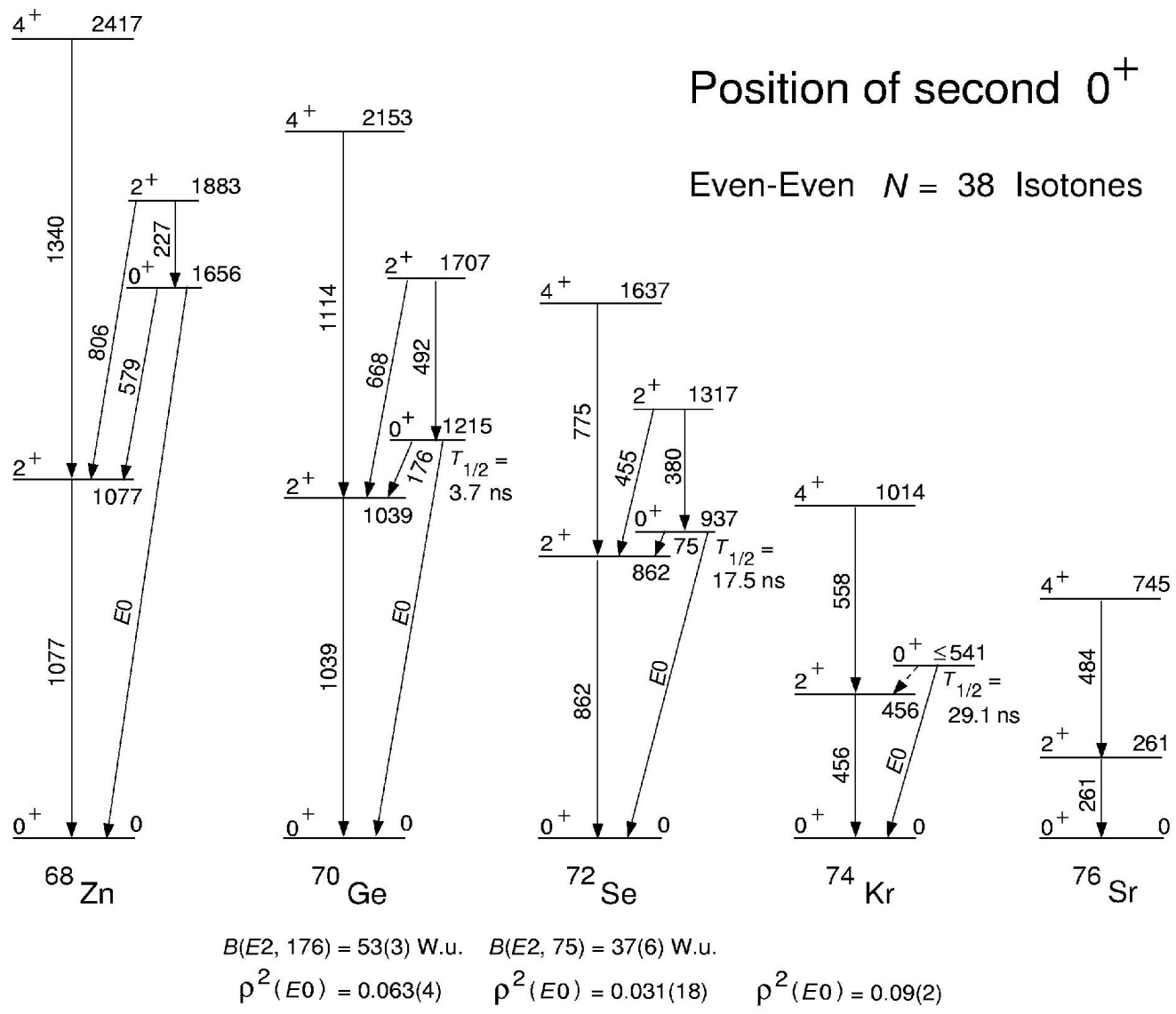

Fig. 7. Excited $0^{+}$states in some neutron-deficient $N=38$ isotones. The experimental $\mathrm{E} 2$ and $\mathrm{E} 0$ transition strengths are given. The data have been taken from: ${ }^{70} \mathrm{Ge}$, Ref. [27]; ${ }^{72} \mathrm{Se}$, Ref. [28]; ${ }^{74} \mathrm{Kr}$, Ref. [25].

\section{Summary and Conclusions}

Modern $\beta$-decay experiments employing multi-Ge detector and scintillator arrays combined with in-flight mass separation of recoils produced via nuclear reactions provide a very sensitive tool for the investigation of low-spin states in nuclei far off the line of stability. This has been demonstrated by the recent results obtained for the highly-fragmented radioactive decay of ${ }^{80} \mathrm{Y} \rightarrow{ }^{80} \mathrm{Sr}$. In general, the new decay data suggest that the low-lying structures of ${ }^{80,84} \mathrm{Sr}$ show many vibration-like features in a potential with modest deformation including candidates for two- and three-phonon multiplets. This vibration-like nature seems to evolve to a more rotational behavior with increasing angular momentum and decreasing neutron number. 


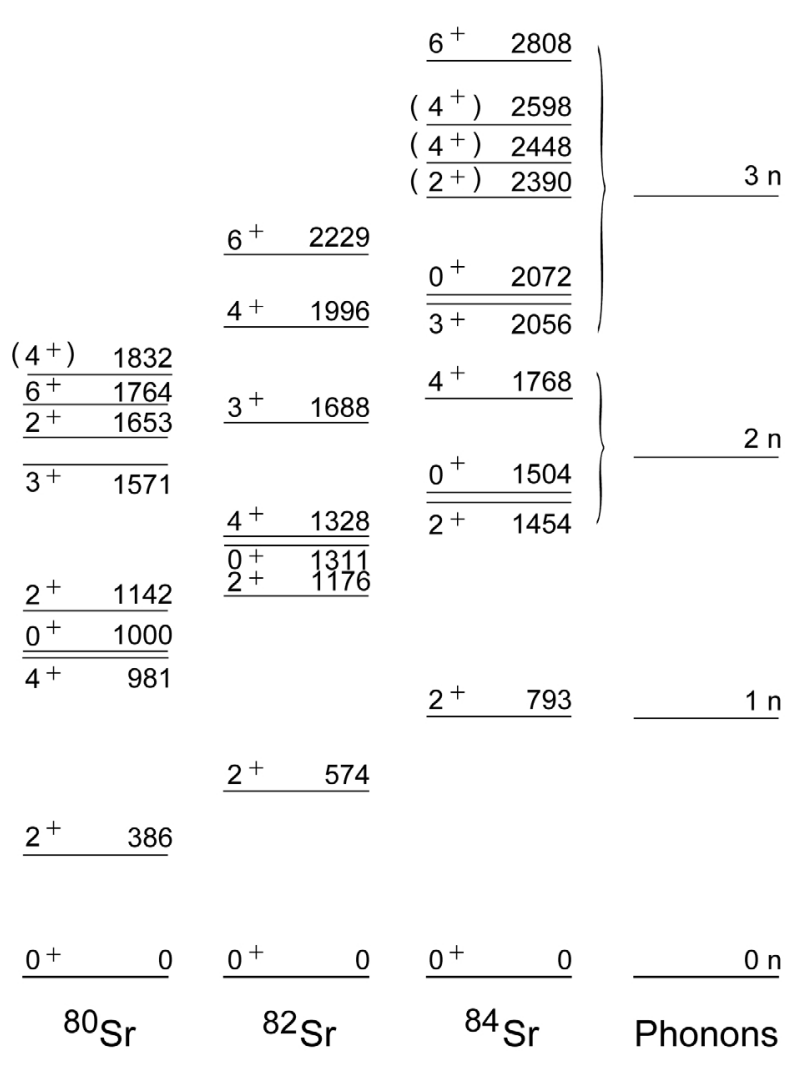

Fig. 8. Low-lying levels in the even-even ${ }^{80,82,84} \mathrm{Sr}$ isotopes. The level energies indicate the vibration-like multiplet structure. For ${ }^{84} \mathrm{Sr}$, the multiple one-phonon energies are given on the right-hand side.

\section{Acknowledgments}

Nuclear physics research at the University of Notre Dame was supported by the National Science Foundation under Grant No. PHY94-02761.

\section{References}

[1] C. J. Lister, B. J. Varley, H. G. Price, and J. W. Olness, Phys. Rev. Lett. 49, 308 (1982).

[2] J. Heese, K. P. Lieb, S. Ulbig, B. Wörmann, J. Billowes, A. A. Chishti, W. Gelletly, C. J. Lister, and B. J. Varley, Phys. Rev. C 41, 603 (1990).

[3] C. J. Lister, P. J. Ennis, A. A. Chishti, B. J. Varley, W. Gelletly, H. G. Price, and A. N. James, Phys. Rev. C 42, R1191 (1990).

[4] W. Nazarewicz, J. Dudek, R. Bengtsson, T. Bengtsson, and I. Ragnarsson, Nucl. Phys. A435, 397 (1985).

[5] D. Galeriu, D. Bucurescu, and M. Ivascu, J. Phys. G 12, 329 (1986).

[6] P. Möller, J. R. Nix, W. D. Myers, and W. J. Swiatecki, At. Data Nucl. Data Tables 59, 185 (1995).

[7] J. L. Wood, K. Heyde, W. Nazarewicz, M. Huyse, and P. van Duppen, Phys. Rep. 215, 101 (1992).

[8] F. Buchinger, E. B. Ramsay, E. Arnold, W. Neu, R. Neugart, K. Wendt, R. E. Silverans, P. Lievens, L. Vermeeren, D. Berdichevsky, R. Fleming, D. W. L. Sprung, and G. Ulm, Phys. Rev. C 41, 2883 (1990).
[9] H. Schatz, A. Aprahamian, J. Görres, M. Wiescher, T. Rauscher, J. F. Rembges, F.-K. Thielemann, B. Pfeiffer, P. Möller, K.-L. Kratz, H. Herndl, B. A. Brown, and H. Rebel, Phys. Rep. 294, 167 (1998).

[10] M. Wiescher, A. Aprahamian, J. Döring, J. Görres, and H. Schatz, Proceedings of the Conf. on Exotic Nuclei and Atomic Masses, AIP Conf. Proc., Vol. 455, Bellaire, Michigan (1998) p. 819.

[11] M. Wiescher, H. Schatz, and A. E. Champagne, Phil. Trans. R. Soc. Lond. A 356, 2105 (1998).

[12] C. N. Davids, B. B. Back, K. Bindra, D. J. Henderson, W. Kutschera, T. Lauritsen, Y. Nagame, P. Sugathan, A. V. Ramayya, and W. B. Walters, Nucl. Instrum. Meth. Phys. Res. B 70, 358 (1992).

[13] J. Döring, H. Schatz, A. Aprahamian, R. C. de Haan, J. Görres, M. Wiescher, W. B. Walters, J. Rikovska, L. T. Brown, C. N. Davids, C. J. Lister, D. Seweryniak, and B. Foy, Phys. Rev. C 57, 1159 (1998).

[14] J. Döring, A. Aprahamian, R. C. de Haan, J. Görres, H. Schatz, M. Wiescher, W. B. Walters, L. T. Brown, C. N. Davids, C. J. Lister, and D. Seweryniak, Phys. Rev. C 59, 59 (1999).

[15] D. M. Moltz, K. S. Toth, F. T. Avignone, N. Noma, B. D. Kern, R. E. Tribble, and J. P. Sullivan, Nucl. Phys. A427, 317 (1984).

[16] R. Iafigliola and J. K. P. Lee, Phys. Rev. C 13, 2075 (1976).

[17] R. B. Firestone and V. S. Shirley, Table of Isotopes, 8th ed., J. Wiley and Sons, Inc., New York (1996).

[18] S. K. Saha, P. E. Haustein, D. E. Alburger, C. J. Lister, J. W. Olness, R. A. Dewberry, and R. A. Naumann, Phys. Rev. C 26, 2654 (1982).

[19] J. Döring, G. Winter, L. Funke, B. Cederwall, F. Lidén, A. Johnson, A. Atac, J. Nyberg, S. J. Freeman, and G. Sletten, NBI Copenhagen, Activity Report 1993, p. 82.

[20] J. Döring et al., (unpublished).

[21] J. B. Ball, J. J. Pinajian, J. S. Larsen, and A. C. Rester, Phys. Rev. C 8, 1438 (1973).

[22] H. Frauenfelder and R. M. Steffen, in Alpha-, Beta- and Gamma-Ray Spectroscopy, Vol. II, K. Siegbahn, ed., NorthHolland Pub. Company, Amsterdam (1965) p. 997.

[23] A. Giannatiempo, A. Nannini, A. Perego, P. Sona, M. J. G. Borge, O. Tengblad, and the Isolde Collaboration, Phys. Rev. C 52, 2444 (1995).

[24] K. Heyde and R. A. Meyer, Phys. Rev. C 37, 2170 (1988).

[25] C. Chandler, P. H. Regan, C. J. Pearson, B. Blank, A. M. Bruce, W. N. Catford, N. Curtis, S. Czajkowski, W. Gelletly, R. Grzywacz, Z. Janas, M. Lewitowicz, C. Marchand, N. A. Orr, R. D. Page, A. Petrovici, A. T. Reed, M. G. Saint-Laurent, S. M. Vincent, R. Wadsworth, D. D. Warner, and J. S. Winfield, Phys. Rev. C 56, R2924 (1997).

[26] R. B. Piercey, J. H. Hamilton, R. Soundranayagam, A. V. Ramayya, C. F. Maguire, X.-J. Sun, Z. Z. Zhao, R. L. Robinson, H. J. Kim, S. Frauendorf, J. Döring, L. Funke, G. Winter, J. Roth, L. Cleemann, J. Eberth, W. Neumann, J. C. Wells, J. Lin, A. C. Rester, and H. K. Carter, Phys. Rev. Lett. 47, 1514 (1981).

[27] A. Passoja, R. Julin, J. Kantele, M. Luontama, and M. Vergnes, Nucl. Phys. A441, 261 (1985).

[28] J. H. Hamilton, A. V. Ramayya, W. T. Pinkston, R. M. Ronningen, G. Garcia-Bermudez, H. K. Carter, R. L. Robinson, H. J. Kim, and R. O. Sayer, Phys. Rev. Lett. 32, 239 (1974).

[29] W. P. Alford, R. E. Anderson, P. A. Batay-Csorba, R. A. Emigh, D. A. Lind, P. A. Smith, and C. D. Zafiratos, Nucl. Phys. A330, 77 (1979).

[30] A. Bohr and B. R. Mottelson, Nuclear Structure, Vol. II, W. A. Benjamin, London (1975) p. 449.

[31] A. Aprahamian, D. S. Brenner, R. F. Casten, R. L. Gill, and A. Piotrowski, Phys. Rev. Lett. 59, 535 (1987). 
About the authors: J. Döring is research scientist at the Gesellschaft für Schwerionenforschung in Darmstadt, Germany, A. Aprahamian is a professor of physics at the University of Notre Dame, and M. Wiescher is the Friedman Professor of Physics at the University of Notre Dame. 